If $\xi_{\nu} \geqq 1$ for each $\nu$, then by reasoning analogous to that of the preceding example, it may be shown, for any set $(a)$, that there is no point $p$ such that $t<p$ implies that $\log S_{t}(a, \xi)$ is convex nor a point $p$ such that $t>p$ implies that $\log S_{t}(a, \xi)$ is concave. Hence Theorem 4 applies to all such functions $\log S_{t}(a, \xi)$. However, for this case the conclusion of the general theorem is weaker than the known result that $\log S_{t}(a, \xi)$ is convex for all positive $t$ and concave for all negative $t .^{2}$

University of California at Los Angeles

${ }^{2}$ See Beckenbach, An inequality of Jensen, Amer. Math. Monthly vol. 53 (1946) pp. 501-505.

\title{
HOMOMORPHISMS ON BANACH SPACES
}

\section{E. MUNROE}

1. Introduction. Let $E$ be a Banach space and $E^{*}$ its conjugate space. Let $G$ be a closed linear subspace of $E$, and let $\Gamma=\left\{f \mid f \in E^{*}\right.$, $f(x)=0$ for $x \in G\}$. Krein and Smulian have shown $\left[4 \text {, Theorem } 12^{\prime}\right]^{1}$ that $G^{*}=E^{*} / \Gamma$ in the sense that the two spaces are algebraically isomorphic and that the usual definitions of norm in the two are equivalent. Noting the algebraic isomorphism, let us look at the topological aspects of this equivalence in a slightly different light. $G^{*}$ being a factor space of $E^{*}$, there is defined a natural homomorphism [5, p. 64] $T\left(E^{*}\right)=G^{*}$. Since they are using the induced topology $[5, \mathrm{p}$. 58] in $E^{*} / \Gamma$, Krein and Smulian prove what is equivalent to the theorem that the transformation $T$ is continuous and open (see [5, Theorem 12]). Stated in this way, incidentally, the result follows immediately from the Hahn-Banach theorem by means of the usual neighborhood argument for continuity and openness.

However, the homomorphism $T\left(E^{*}\right)=G^{*}$ suggests other topological questions the answers to which are not quite so obvious. Specifically, what are the topological properties of $T$ when $E^{*}$ and $G^{*}$ are given topologies other than their norm topologies?

The conjugate to a Banach space may be topologized in any one of several well known ways. The most common such topologies are the norm, weak, weak*, bounded weak and bounded weak*. We shall

Presented to the Society, October 25, 1947; received by the editors September 10, 1947.

${ }^{1}$ Numbers in brackets refer to the bibliography at the end of the paper. 
use these terms in connection with properties of $T$ to designate the property mentioned when both $E^{*}$ and $G^{*}$ have the topology mentioned. For example, the statement, " $T$ is weak* continuous," means that $T$ is continuous when $E^{*}$ is given its weak* topology and $G^{*}$ is given its weak* topology.

2. Notes on weak topologies. Before proceeding, let us review the definitions and some of the properties of the various weak topologies of a conjugate space $E^{*}$.

Definition 2.1. A directed set $\left\{f_{\alpha}\right\} \subset E^{*}$ has $f \in E^{*}$ as its weak* limit provided $\lim _{\alpha} f_{\alpha}(x)=f(x)$ for every $x \in E$. This topology is described by the neighborhoods of the origin $U(\sigma, \epsilon)=\{f|| f(x) \mid<\epsilon$ for $x \in \sigma\}$ where $\sigma$ is a finite set in $E$ and $\epsilon>0$.

Definition 2.2. A directed set $\left\{f_{\alpha}\right\} \subset E^{*}$ is weakly convergent to $f \in E^{*}$ provided $\lim _{\alpha} X\left(f_{\alpha}\right)=X(f)$ for every $X \in E^{* *}$. Neighborhoods of the origin for this topology are $U(\Sigma, \epsilon)=\{f|| X(f) \mid<\epsilon$ for $X \in \Sigma\}$ where $\Sigma$ is a finite set in $E^{* *}$ and $\epsilon>0$.

Bounded weak* and bounded weak topologies are obtained from definitions 2.1 and 2.2 respectively, if the provision is added that $\left\{f_{\alpha}\right\}$ converges to $f$ only if there is a number $K$ such that $\left\|f_{\alpha}\right\| \leqq K$ for every $\alpha$.

For sequences, the notions of weak $^{*}$ and bounded weak* convergence are equivalent, as are those of weak and bounded weak convergence, because convergent sequences are necessarily bounded $[2$, pp. 80, 123]. However, this is not true for directed sets in general. Every conjugate space is boundedly weak* complete; but Alaoglu has shown $[1$, p. 254$]$ that if $E^{*}$ is infinite-dimensional, it is not weak* complete. An example similar to Alaoglu's shows that if $E^{*}$ is infinitedimensional, it contains a directed set weak* convergent to the null functional for which no bounded subset has the null functional as a limit point.

Let $\left\{x_{1}, x_{2}, x_{3}, \ldots\right\}$ be a sequence of linearly independent elements of $E$ with $\left\|x_{i}\right\|=1$ for each $i$. Let the finite-dimensional subspaces of $E$ be partially ordered by means of the inclusion relationship. These subspaces $\left\{G_{\alpha}\right\}$ then form a directed set. We form a corresponding directed set $\left\{f_{\alpha}\right\} \subset E^{*}$ as follows: if $G_{\alpha}$ is $n$-dimensional, at least one of the points $x_{1}, x_{2}, \cdots, x_{n+1}$ lies outside it; hence $\left[2\right.$, p. 57] there is a functional $f_{\alpha}$ which is zero on $G_{\alpha}$ and equal to $n$ at this point. Thus $\left\|f_{\alpha}\right\| \geqq n$. Clearly $\lim _{\alpha} f_{\alpha}(x)=0$ for every $x \in E$, but the neighborhood $U\left(\sigma_{n+1}, 1\right)-\sigma_{n+1}=\left\{x_{1}, x_{2}, \cdots, x_{n+1}\right\}$-contains no member of $\left\{f_{\alpha}\right\}$ with norm less than $n$.

An analogous construction will demonstrate the non-equivalence 
of weak and bounded weak convergence in infinite-dimensional spaces.

With regard to closure of linear subspaces, however, the picture is slightly different; and it is here that the weak* topology comes into its own. For linear subspaces of $E^{*}$ the notions of weak* and bounded weak* closure are equivalent. Furthermore, they are equivalent [1, Theorem 1.4] to regular closure, defined by Banach as follows:

Definition 2.3. A linear subspace $\Gamma$ of $E^{*}$ is regularly closed provided to every $f \in E^{*}-\Gamma$ there corresponds an $x \in E$ such that $f(x)=1$ and $g(x)=0$ for every $g \in \Gamma$.

Equivalence of weak* and bounded weak* closure for linear subspaces seems to be known [3, p. 52]. The proof is contained in the work of Alaoglu [1, p. 256], but the result is not noted there. Alaoglu's proof that regular closure implies bounded weak* closure makes no use of boundedness. Thus weak* closure may be inserted between regular and bounded weak* closure in his chain of implications. It might be remarked in passing that in his discussion of these relationships for the case of separable $E$, Banach occasionally speaks of sets with no qualification when he means linear sets [2, Theorem 5, p. 121 and summary of results, p. 126].

Finally, we recall one of the most important properties of the weak* topology:

Theorem 2.4 (Alaoglu [1, Theorem 1.3]). The unit sphere in $E^{*}$ is bicompact in the weak* topology.

3. Topological properties of the homomorphism $T$. Returning now to the natural homomorphism of $E^{*}$ onto $G^{*}$, we first investigate the questions of continuity and openness. Continuity is obvious in each case: hence we state without proof:

THEOREM 3.1. T is norm continuous, weakly continuous, boundedly weakly continuous, weak* continuous and boundedly weak* continuous.

As noted in the introduction, due to the work of Krein and Smulian, we may add:

\section{THEOREM 3.2. $T$ is norm open.}

For use in subsequent proofs, we introduce the operation $\oplus$ to denote the direct sum of sets in $E$ (or $E^{*}$, as the case may be). $A \oplus B$ $=\{x \mid x=a+b, a \in A, b \in B\}$.

THEOREM 3.3. $T$ is weak* open.

Let $U(\sigma, \epsilon)$ be any weak* neighborhood in $E^{*}$. To prove that $T$ is 
weak* open, we must show $[5$, p. 59] that $T[U(\sigma, \epsilon)]$ contains a weak* neighborhood in $G^{*}$. Specifically, we shall construct a set $\tau \subset G$ such that $T[U(\sigma, \epsilon)] \supset U(\tau, \epsilon)$. If, as in the introduction, we denote the denominator subspace by $\Gamma$, this means that having constructed $\tau$, we must show that to every $f \in U(\tau, \epsilon)$ there corresponds a $g \in \Gamma$ such that $f+g \in U(\sigma, \epsilon)$.

The set $\tau$ is constructed as follows: $\sigma$ being a finite set, it is contained in a finite-dimensional subspace $S$ of $E$. $S$ being finite-dimensional, the factor space $(S \oplus G) / G$ is finite-dimensional and therefore has a finite basis. Let $\left\{x_{1}, x_{2}, \cdots, x_{h}\right\}$ be a set of unit elements of $S$, each chosen from a different member of the basis for $(S \oplus G) / G$. The following are then easily verified:

(1) If $x \in \sigma, x=y_{x}+\sum_{j=1}^{k} a_{j} x_{j}$, where $y_{x} \in G$ and the $a_{j}$ are real coefficients (depending, of course, on $x$ ).

(2) For each $i(i=1,2, \cdots, k), x_{i}$ lies outside the closed linear subspace $G \oplus S_{i}$, where $S_{i}$ is the subspace generated by $x_{1}, x_{2}, \cdots, x_{i-1}$, $x_{i+1}, \cdots, x_{k}$.

We now define $\tau$ to be the set of all $y_{x}$ (see (1)) for $x \in \sigma$. Now given any $f \in E^{*}$, it follows from (2) and a familiar property of closed linear subspaces $\left[2\right.$, p. 57] that there exists $g_{i} \in E^{*}$ such that $g_{i}(y)=0$ for $y \in G \oplus S_{i}$ and $g_{i}\left(x_{i}\right)=-f\left(x_{i}\right)$. We let $g=\sum_{i=1}^{k} g_{i}$; then $g \in \Gamma$, and the expansion in (1) immediately yields the result that if $x \in \sigma, f(x)+g(x)$ $=f\left(y_{x}\right)$. Hence $f \in U(\tau, \epsilon)$ implies $f+g \in U(\sigma, \epsilon)$. Q.E.D.

\section{THEOREM 3.4. T is weakly open.}

Krein and Smulian have shown [4, Theorem 13] that $G^{* *}$ is a subspace of $E^{* *}$. As a matter of fact, it is a regularly closed subspace, as is seen immediately from the construction they give for it and a remark of Banach's [2, p. 117]. Armed with this information, we can reverse the roles of elements and functionals and proceed as in the proof of Theorem 3.3. The only difference is that we use regular closure instead of Banach's lemma to guarantee the existence of the $g_{i}$. This calls for regular closure of subspaces of the form $G^{* *} \oplus S_{i}$ where $S_{i}$ is finite-dimensional. This follows immediately from regular closure of $G^{* *}$ if we replace regular closure by weak* closure and consider limits of directed sets.

There remain the questions of bounded weak and bounded weak* openness for $T$. The construction given in the proof of Theorem 3.3 does not answer these. The norm of each $g_{i}$ may be as large as the norm of $f$; hence the bound on norms might conceivably have to be multiplied by the number of elements in $\sigma$. We do not have an example handy to show that this unfavorable situation can actually oc- 
cur; but because of the equivalence of the bounded and unbounded closures for linear subspaces, this point can frequently be by-passed (see Theorem 3.5 below).

The transformation $T$ is not closed with respect to any of the topologies considered. This is shown by a very simple example. Let $E^{*}$ be Euclidian 2-space, and let $\Gamma$ be the $y$-axis. Then $G^{*}$ is the $x$-axis and $T$ is the orthogonal projection transformation. Consider the closed set $A=\{(x, y) \mid x>0, y>0, x y \geqq 1\}$. Then $T(A)=\{x \mid x>0\}$ which is not closed. The five topologies are equivalent here; so this example answers all five questions. Note that the set $A$ above is convex; so we cannot say even that $T$ is closed for convex sets.

The theorems on openness of $T$ may be used to prove closure by taking complements, provided we first take complete inverse images. Now $T^{-1}[T(\Delta)]=\Delta \oplus \Gamma$; thus we can show that $T(\Delta)$ is closed by showing that

(A) $\Delta \oplus \Gamma$ is closed, and

(B) the topology used is one of those discussed in Theorems 3.2, 3.3 and 3.4 or is equivalent to one of these for sets of the type $\Delta \oplus \Gamma$.

Using this technique, we arrive very easily at the following results:

THEOREM 3.5. "1. For linear subspaces, $T$ is (a) weak* closed, (b) boundedly weak* closed.

2. For compact sets, $T$ is closed with respect to each of the five topologies.

3. For bounded sets, $T$ is (a) weak* closed, (b) boundedly weak* closed.

Statements 1(a) and 1(b) are equivalent. For 1(b), the condition (A) is satisfied because unit spheres are bicompact (Theorem 2.4), and the direct sum of two bicompact sets is bicompact, hence closed. Turning now to $1(\mathrm{a})$, we see that Theorem 3.3 applies to this topology. Thus condition (B) is also satisfied.

To prove 2, we note that the direct sum of a compact set and a closed set is closed. The openness theorems then dispose of three cases immediately. To cover the cases of the bounded topologies we note that for sets $\Delta \oplus \Gamma$ where $\Delta$ is compact, every limit point is the limit of a bounded subset. Every point is of the form $f+g$ where $f \in \Delta$ and $g \in \Gamma$. Since $\Delta$ is compact (henced bounded), the $f$ 's are automatically bounded; and since $\Gamma$ is linear, the $g$ 's may be replaced by a bounded subset.

Finally, we note that 3 reduces to 2 by means of Theorem 2.4. In connection with 3 , we might point out that weak* closure of $T$ for bounded sets is quite a different thing from bounded weak* closure 
of $T$. The natural homomorphism has the first of these properties, but not the second.

The most obvious unanswered questions are those of norm and weak closure of $T$ for linear subspaces. By means of Theorems 3.2 and 3.4, these are reduced to questions of closure of direct sums; but the compactness arguments of Theorem 3.5 must be replaced by others.

\section{BIBLIOGRAPHY}

1. L. Alaoglu, Weak topologies of normal linear spaces, Ann. of Math. vol. 41 (1940) pp. 252-267.

2. S. Banach, Theorie des operations linéaires, Warsaw, 1932.

3. W. F. Eberlein, Weak compactness in Banach spaces I, Proc. Nat. Acad. Sci. U. S. A. vol. 33 (1947) pp. 51-53.

4. M. Krein, and V. Smulian, On regularly convex sets in the space conjugate to a Banach space, Ann. of Math. vol. 41 (1940) pp. 556-583.

5. L. Pontrjagin, Topological groups, Princeton, 1939.

UNIVERSITY OF ILLINOIS 